\title{
Erratum: Antibody production in rabbits administered Freund's complete adjuvant and carprofen concurrently
}

Joanna E. Fishback, MPH, DVM, DACVPM, Sabrina M. Stronsky, MS, Catherine A. Green, BS, ALAT, Krystal D. Bean, DVM, DACLAM \& Jeffrey W. Froude, $P h D$

Lab Anim. (NY) 45, 63-66 (2016); corrected after print 27 January 2016.

In the version of this article published in print, the affiliation for the five authors was omitted. The error was corrected in the HTML and PDF versions of the article before online publication. 